ARTICLE

\title{
GPCR voltage dependence controls neuronal plasticity and behavior
}

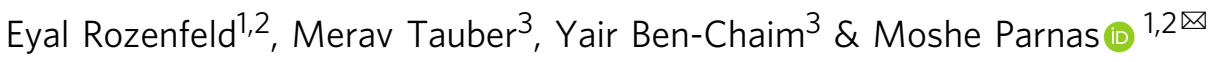

G-protein coupled receptors (GPCRs) play a paramount role in diverse brain functions. Almost 20 years ago, GPCR activity was shown to be regulated by membrane potential in vitro, but whether the voltage dependence of GPCRs contributes to neuronal coding and behavioral output under physiological conditions in vivo has never been demonstrated. Here we show that muscarinic GPCR mediated neuronal potentiation in vivo is voltage dependent. This voltage dependent potentiation is abolished in mutant animals expressing a voltage independent receptor. Depolarization alone, without a muscarinic agonist, results in a nicotinic ionotropic receptor potentiation that is mediated by muscarinic receptor voltage dependency. Finally, muscarinic receptor voltage independence causes a strong behavioral effect of increased odor habituation. Together, this study identifies a physiological role for the voltage dependency of GPCRs by demonstrating crucial involvement of GPCR voltage dependence in neuronal plasticity and behavior. Thus, this study suggests that GPCR voltage dependency plays a role in many diverse neuronal functions including learning and memory.

\footnotetext{
${ }^{1}$ Department of Physiology and Pharmacology, Sackler School of Medicine, Tel Aviv University, Tel Aviv 69978, Israel. ${ }^{2}$ Sagol School of Neuroscience, Tel Aviv University, Tel Aviv 69978, Israel. ${ }^{3}$ Department of Natural and Life Sciences, The Open University of Israel, Ra'anana 43107, Israel.

凶email: mparnas@tauex.tau.ac.il
} 
G protein coupled receptors (GPCRs) comprise the largest known family of transmembrane receptors, and are present in all life forms ${ }^{1}$. Over $90 \%$ of non-sensory GPCRs are expressed in the brain, where they mediate responses to various biologically active molecules including acetylcholine, glutamate, dopamine, noradrenaline, serotonin, histamine, GABA, peptides, lipid-derived products, and also to mechanical stimuli $^{2,3}$. As such, they play a paramount role in diverse brain functions, for example, vision, taste, olfaction, behavior regulation, neuromodulation, and regulation of the immune system among others. GPCRs thus mediate a large variety of brain and body functions and are critical for normal brain function ${ }^{4-6}$.

GPCRs are activated by binding of specific agonists. They then interact with GTP-binding proteins (G-proteins) and mediate their effect via a range of second messenger signaling pathways in the cell ${ }^{4-6}$. Almost 20 years ago, the activity level, as well as agonist binding affinity, of several GPCRs were shown to be regulated by membrane potential in vitro ${ }^{7-15}$. For example, the activity of cholinergic $M_{2}$ muscarinic receptors $\left(M_{2} R\right)^{10}$, metabotropic glutamate receptors ${ }^{14}$, and $\alpha 2$ adrenergic receptors ${ }^{12}$ are reduced by depolarization. In contrast, the activity of $M_{1}$ muscarinic receptors $\left(\mathrm{M}_{1} \mathrm{R}\right)^{10}$ and mGluRla glutamate receptors ${ }^{14}$ are increased by depolarization. GPCR voltage dependency is about an order of magnitude weaker than that of voltage-gated channels ${ }^{16}$, and GPCRs lack the type of voltage sensor composed of positively charged residues, seen in voltage-gated channels ${ }^{11}$.

Despite several decades of research into the functions of GPCRs, it is still not clear whether GPCR voltage dependency plays a role in vivo under physiological conditions or contributes to neuronal coding and behavioral output. Although GPRC voltage dependence was shown to control synaptic release initiation and duration in vitro ${ }^{17-22}$, there is no evidence that these small changes in the duration of synaptic release affect neuronal computation or behavioral output, especially in the background of noisy neural activity. Thus, the question of whether GPCR voltage dependency plays a role in vivo under physiological conditions and contributes to neuronal coding and behavioral output remains open.

Currently, the only known voltage sensor of GPCRs is for muscarinic receptors. The Drosophila olfactory system is cholinergic and has high expression levels of muscarinic receptors ${ }^{23}$. Only two Drosophila muscarinic receptors are expressed in the fly brain: a $\mathrm{G}_{\mathrm{q}}$ coupled Drosophila muscarinic type A receptor (mAChR-A, homologous to $\mathrm{M}_{1} \mathrm{R}$ ); and a $\mathrm{G}_{\mathrm{i} / \mathrm{o}}$ coupled Drosophila muscarinic type $\mathrm{B}$ receptor (mAChR-B, homologous to $\left.\mathrm{M}_{2} \mathrm{R}\right)^{24,25}$. Although the Drosophila genome contains a third Drosophila muscarinic type $\mathrm{C}$ receptor, its expression level in the brain is negligible 26,27 . As a result, manipulation of Drosophila muscarinic receptors results in profound physiological and behavioral effects ${ }^{23,28}$, which makes, the Drosophila olfactory system well suited to examine whether GPCR voltage dependence affects physiological processes and behavior.

Odors activate Drosophila cholinergic olfactory receptor neurons (ORNs) that are located in the antennae and maxillary palps. Each ORN expresses a single odorant receptor gene ${ }^{29,30}$. ORNs expressing the same receptor send their axons to a single glomerulus in the antennal lobe (AL, homologous to the mammalian olfactory bulb) ${ }^{31-33}$. Second-order excitatory cholinergic projection neurons (ePNs) send their dendrites to a single glomerulus and serve as the primary output channel of the $\mathrm{AL}^{34}$. The $\mathrm{AL}$ also contains multi glomeruli inhibitory GABAergic and glutamatergic local neurons (iLNs) ${ }^{35,36}$ that receive input from ORNs and PNs.

We recently demonstrated that $\mathrm{mAChR}-\mathrm{A}$ is mainly localized to a subpopulation of GABAergic iLNs where it induces shortterm potentiation of iLNs ${ }^{23}$. Knockdown of mAChR-A in iLNs also causes changes of odor valence ${ }^{23}$. In addition to odor valence, iLN activity is required for odor habituation, sustained odor responses, regulating gain control, signal separation, and enhancement of interglomerular contrast ${ }^{36-43}$.

Here we show that the Drosophila mAChR-A, which diverged from the mammalian muscarinic receptors over 700 million years ago $^{24}$ is also voltage dependent. We use Clustered Regularly Interspaced Short Palindromic Repeats (CRISPR/Cas9) to generate a fly strain with two point mutations that abolish the mAChR-A voltage dependence while retaining normal maximal activity. This enables us to demonstrate that wt mAChR-A induced post-tetanic potentiation (PTP) is voltage dependent and that this dependency is abolished in mutant flies expressing a voltage independent mAChR-A. Even more striking is that depolarization alone, without any agonist, causes a potentiation of nicotinic receptors in iLNs which is mediated by mAChR-A. In addition, the generation of a voltage-independent mAChR-A results in a pronounced behavioral effect of increased odor habituation, which was localized to iLNs. Taken together, this study provides a demonstration of a physiological role for the voltage dependency of GPCRs, and may serve as a paradigm shift in our understanding of neural function and drug discovery.

\section{Results}

The Drosophila mAChR-A GPCR is voltage dependent. The Drosophila mAChR-A has overall low sequence homology to its mammalian homolog $\mathrm{M}_{1} \mathrm{R}^{24}$. We therefore first examined the voltage dependency of $\mathrm{mAChR}-\mathrm{A}$ expressed in Xenopus oocytes. As for $\mathrm{M}_{1} \mathrm{R}$, depolarization $(+40 \mathrm{mV})$ increased $\mathrm{mAChR}-\mathrm{A}$ activity as indicated by a leftward shift in the mAChR-A dose-response curve compared to the curve obtained by hyperpolarization $(-80 \mathrm{mV}$, Fig. 1A). Thus, when the membrane potential is depolarized, $\mathrm{mAChR}-\mathrm{A}$ is in a high activity state, and when the membrane potential is hyperpolarized, it is in a low activity state. In order to isolate the effects of the voltage dependency of mAChR-A from other voltage-related effects, we sought to generate a voltage-independent mAChR-A. Direct gating current measurements revealed that the voltage sensor of mammalian muscarinic receptors consists of three tyrosine residues (Supplementary Fig. 1A) ${ }^{44}$. Replacing these residues with the electrically neutral amino acid, alanine, abolished both the gating currents and the voltage-dependent activity of $\mathrm{M}_{2} \mathrm{R}^{44}$. Attempts at replacing these residues in $\mathrm{mAChR}-\mathrm{A}$ resulted in impaired receptor activity unsuitable for further experiments (Supplementary Fig. 1).

Since these attempts to generate a voltage-independent receptor proved unsuccessful, we sought a different approach. The $\mathrm{N}$ - and $\mathrm{C}$ - terminals of the third intracellular loop of $\mathrm{M}_{1} \mathrm{R}$ and $\mathrm{M}_{2} \mathrm{R}$ are required for $\mathrm{G}$-protein coupling ${ }^{45,46}$, and replacing the $M_{2} R$ N-terminal residues KKDKK with the $M_{1} R$ N-terminal residues ELAAL was shown to abolish the $\mathrm{M}_{2} \mathrm{R}$ voltage dependence while retaining receptor function ${ }^{11}$. Since the homologous motif in mAChR-A is KDLPN (Supplementary Fig. 1C), we replaced aspartic acid at position 301 and proline at position 303 with lysine (D301K, P303K) to generate mAChR-A with a final motif sequence of KKLKN. This modified receptor that we term mAChR-A-KK, possesses voltage-independent activity resembling that of $\mathrm{mAChR}-\mathrm{A}$ in the high activity state, i.e. when depolarized (Fig. 1B). Importantly, the response of mAChR-A-KK to saturating levels of ACh resembles that of $w t$ mAChR-A at the two membrane potentials tested (Fig. 1D), indicating that the overall activity of mAChR-A-KK is not impaired. Similarly, measuring endogenous $\mathrm{Ca}^{2+}$ activated $\mathrm{Cl}^{-}$ channels, activated by $\mathrm{G}_{\mathrm{q}}$ protein ${ }^{10}$ yielded the same results (Supplementary Fig. 2A, B). We also verified that receptor 

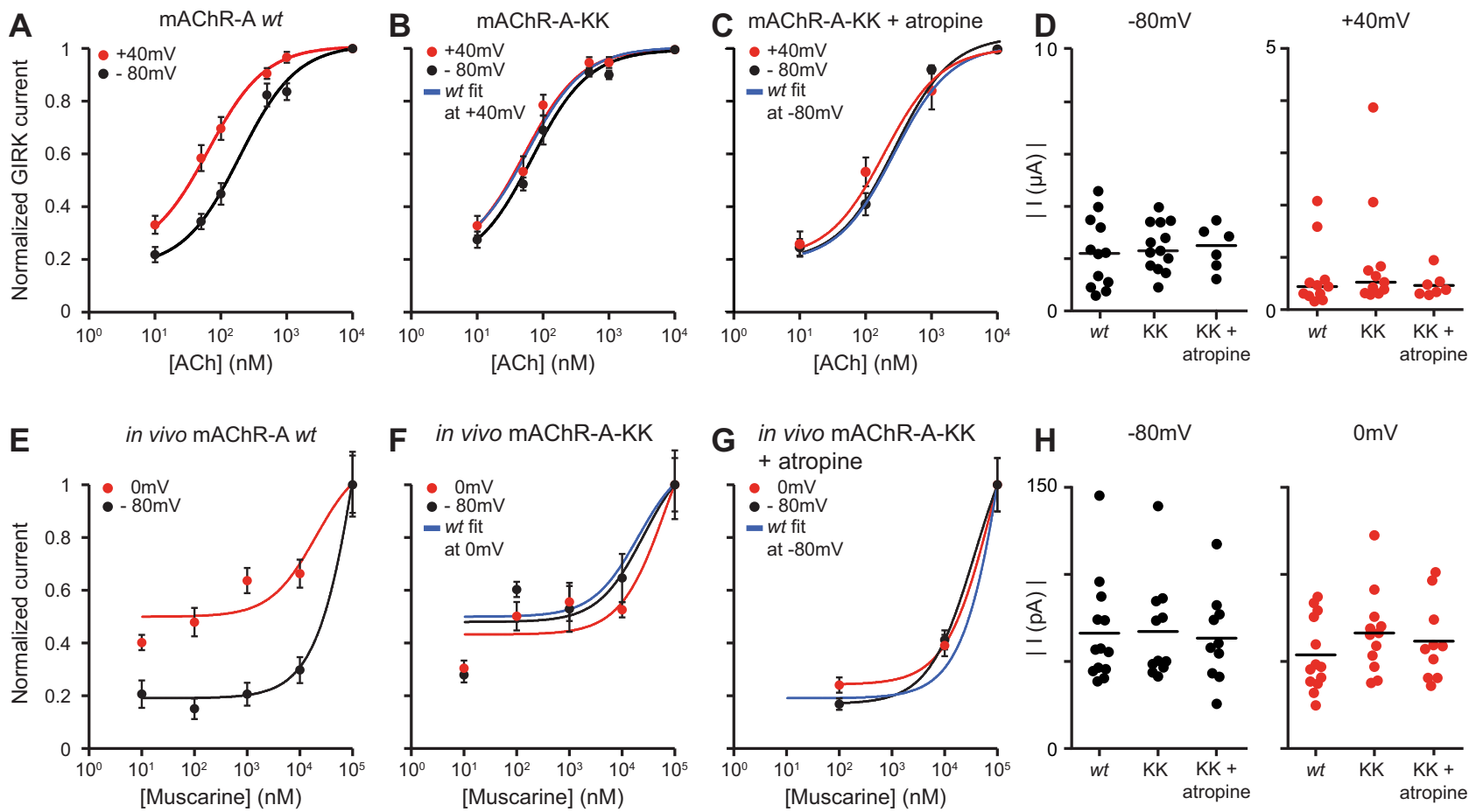

$0 \mathrm{mV}$

Fig. 1 in vitro and in vivo voltage dependence of $\mathbf{m A C h R - A . ~ A ~ D o s e - r e s p o n s e ~ c u r v e s ~ f o r ~ w t ~ m A C h R - A - e v o k e d ~ G I R K ~ c u r r e n t s ~ i n ~ o o c y t e s ~ a t ~}-80 \mathrm{mV}$ (black; $n=24$ ) and $+40 \mathrm{mV}$ (red; $n=17$ ). The curve of the depolarized membrane potential is shifted to the left indicating a higher activity state. B Doseresponse curves for $\mathrm{mAChR-A-KK-evoked} \mathrm{GIRK}$ currents in oocytes at $-80 \mathrm{mV}$ (black; $n=23$ ) and $+40 \mathrm{mV}$ (red; $n=19$ ). $\mathrm{mAChR}-\mathrm{A}$-KK is in the high activity state as indicated by the overlap with the dose-response curve of wt $\mathrm{mAChR}-\mathrm{A}$ at $+40 \mathrm{mV}$ (blue line). C Dose-response curves for $\mathrm{mAChR}-\mathrm{A}-\mathrm{KK}$ evoked GIRK currents in oocytes at $-80 \mathrm{mV}$ (black; $n=6$ ) and $+40 \mathrm{mV}$ (red; $n=7$ ) in the presence of $1 \mathrm{nM}$ of atropine. Atropine shifts $\mathrm{mAChR-A-KK}$ to the lower activity state as indicated by the overlap with the curve of wt mAChR-A at $-80 \mathrm{mV}$ (blue line). D Absolute current values of wt $\mathrm{mAChR}$ - , $\mathrm{mAChR}-\mathrm{A}-\mathrm{KK}$, and mAChR-A-KK in the presence of $1 \mathrm{nM}$ atropine, for ACh evoked currents in oocytes at $-80 \mathrm{mV}$ (left, black; $n=12,13$, and 6 , respectively) and $+40 \mathrm{mV}$ (right, red; $n=11,11$ and 7 , respectively) elicited by a saturating level of $A C h$. Data are un-normalized values of panels $\mathbf{A}$-C at $10^{4} \mathrm{nM}$ ACh. E In vivo dose-response curves for wt flies of muscarine evoked currents in iLNs at $-80 \mathrm{mV}\left(\mathrm{black} ; 10^{1} \mathrm{nM} n=11,10^{2} \mathrm{nM} n=9,10^{3} \mathrm{nM}\right.$ $n=9,10^{4} \mathrm{nM} n=14,10^{5} \mathrm{nM} n=13$ ) and $0 \mathrm{mV}$ (red; $10^{1} \mathrm{nM} n=9,10^{2} \mathrm{nM} n=10,10^{3} \mathrm{nM} n=10,10^{4} \mathrm{nM} n=14,10^{5} \mathrm{nM} n=13$ ). F In vivo dose-response curves for $\mathrm{mAChR}-\mathrm{A}-\mathrm{KK}$ fly strain of muscarine evoked currents in iLNs at $-80 \mathrm{mV}$ (black; $10^{1} \mathrm{nM} n=11,10^{2} \mathrm{nM} n=13,10^{3} \mathrm{nM} n=11,10^{4} \mathrm{nM} n=11,10^{5}$ $\mathrm{nM} n=11$ ) and $0 \mathrm{mV}$ (red; $10^{1} \mathrm{nM} n=11,10^{2} \mathrm{nM} n=18,10^{3} \mathrm{nM} n=18,10^{4} \mathrm{nM} n=12,10^{5} \mathrm{nM} n=12$ ). G In vivo dose-response curves for $\mathrm{mAChR}-\mathrm{A}-\mathrm{KK}$ fly strain of muscarine evoked currents in iLNs at $-80 \mathrm{mV}$ (black; $10^{2} \mathrm{nM} \mathrm{n}=11,10^{4} \mathrm{nM} n=12,10^{5} \mathrm{nM} n=10$ ) and $0 \mathrm{mV}\left(\mathrm{red} ; 10^{2} \mathrm{nM} n=12,10^{4} \mathrm{nM}\right.$ $n=11,10^{5} \mathrm{nM} n=10$ ) in the presence of $1 \mu \mathrm{M}$ atropine. Atropine shifts mAChR-A-KK to the lower activity state as indicated by the overlap with the dose-response curve of wt $\mathrm{mAChR}-\mathrm{A}$ at $-80 \mathrm{mV}$ (blue line). H Absolute current values of in vivo wt ( $-80 \mathrm{mV}, n=13,0 \mathrm{mV}, n=13), \mathrm{mAChR}-\mathrm{A}-\mathrm{KK}$ $(-80 \mathrm{mV}, n=11,0 \mathrm{mV}, n=12)$, and $\mathrm{mAChR}-\mathrm{A}-\mathrm{KK}$ flies in the presence of $1 \mu \mathrm{M}$ atropine $(-80 \mathrm{mV}, n=10,0 \mathrm{mV}, n=10)$, for muscarine evoked currents in iLNs at $-80 \mathrm{mV}$ (left, black) and $0 \mathrm{mV}$ (right, red) elicited by a saturating level of muscarine. Each dot represents a single fly. Data are un-normalized values of panels E-G at $10^{5} \mathrm{nM}$ muscarine. For all panels, error bars represent the standard error of the mean (SEM). Source data are provided as a Source Data file.

recruitment of the downstream $G_{q}$ pathway was not impaired (Supplementary Fig. 2B, C) and that receptor desensitization by $\beta$-arrestin and $G$ protein-coupled receptor kinase 3 (GRK3) coexpressed in the oocyte was not affected in mAChR-A-KK (Supplementary Fig. 3). In order to obtain the voltageindependent mAChR-A-KK in the low activity state, we applied a low concentration of the competitive antagonist, atropine. In contrast to the situation in mammalian muscarinic receptors, atropine is specific for mAChR-A in flies and does not affect mAChR-B, allowing us to exploit this method also in vivo ${ }^{25}$. Application of atropine shifted the mAChR-A-KK dose-response curve to the right, so that it overlapped with the activity of $w t$ mAChR-A in the low activity state (Fig. 1C). Importantly, even in the presence of atropine, the response of mAChR-A-KK to saturating levels of acetylcholine (ACh) was not different from that of the $w t$ mAChR-A at any of the tested membrane potentials (Fig. 1D). Thus, by using two complementary approaches of pharmacology and gene editing, we generated a voltage-independent $\mathrm{mAChR}-\mathrm{A}-\mathrm{KK}$ receptor that has normal maximal activity and can reside in a high or low activity state.

Even though the voltage dependence of GPCRs has been known for years, the physiological relevance to in vivo processes remains unknown. To examine the effects of mAChR-A voltage dependence on physiological processes and behavioral output, we used CRISPR to generate a fly strain that has the two-point mutations (i.e. D301K, P303K) that produce mAChR-A-KK. We have previously shown that the muscarinic agonist muscarine causes an increase in $\mathrm{Ca}^{2+}$ levels in iLNs ${ }^{23}$, probably by activation of $\mathrm{Ca}^{2+}$ channels ${ }^{47}$, resulting in an inward current flow. We now used these muscarine-induced currents to verify that wt mAChRA is voltage dependent in vivo and to examine whether the $\mathrm{D} 301 \mathrm{~K}, \mathrm{P} 303 \mathrm{~K}$ point mutations generate a voltage-independent mAChR-A in vivo. Similar to the results obtained with oocytes, in vivo mAChR-A activity was voltage dependent in $w t$ flies and voltage independent in the mAChR-A-KK fly strain (Fig. 1E-G). In addition, the $w t$ and mutant receptors responded in the same 
A
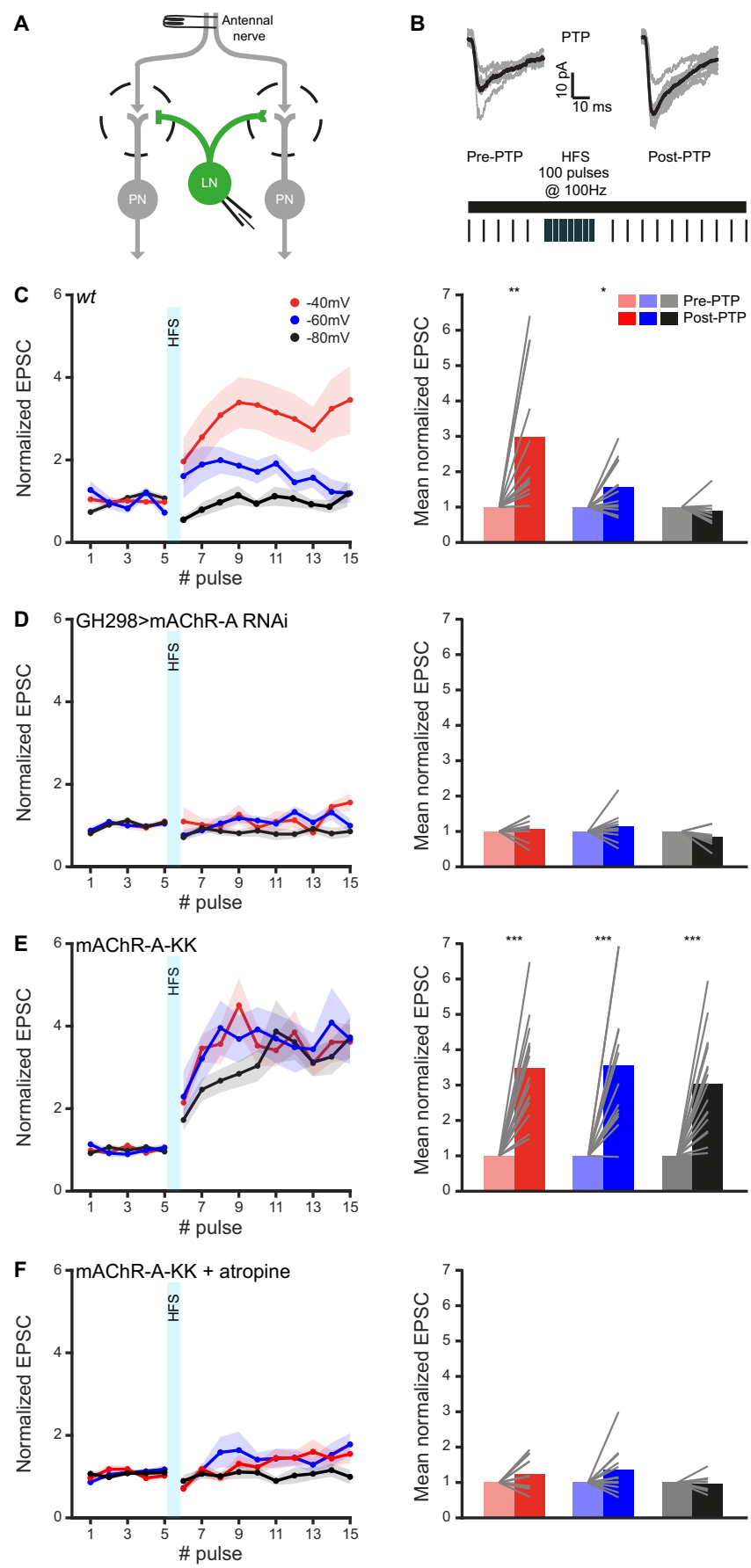

way to saturating levels of muscarine at all the measured membrane potentials, indicating that mAChR-A-KK activity is not impaired in vivo (Fig. 1H).

mAChR-A voltage dependency controls neuronal potentiation. We have recently reported that $\mathrm{mAChR}$-A contributes to shortterm potentiation in iLNs labeled by the GH298-GAL4 driver line ${ }^{23}$. Having demonstrated the voltage dependency of mAChRA activity, it was now interesting to examine whether the iLN potentiation is similarly voltage dependent. To this end, we used a PTP protocol of 100 antennal nerve stimulations at $100 \mathrm{~Hz}$ to ORNs that are presynaptic to iLNs (Fig. 2A, B). Postsynaptic iLNs were held at either $-40 \mathrm{mV},-60 \mathrm{mV}$, or $-80 \mathrm{mV}$ during the PTP phase, and then returned to their resting membrane potential $(-60 \mathrm{mV})$. Surprisingly, the results indicated that while
Fig. 2 mAChR-A induced potentiation is voltage dependent. A

Experimental configuration. ORNs axons were stimulated at varying frequencies and the iLN post-synaptic evoked currents were measured using in vivo whole-cell voltage clamp. B Post-Tetanic Potentiation (PTP) protocol. Representative single fly traces (gray) of excitatory postsynaptic current (EPSCs) obtained before (left) and after (right) the PTP protocol. The mean EPSC is shown in black. C Left, normalized EPSC before and after PTP obtained in wt flies for holding potential of $-80 \mathrm{mV}$ (black; $n=10$ ), $-60 \mathrm{mV}$ (blue; $n=13$ ), and $-40 \mathrm{mV}$ (red; $n=12$ ). Right, mean normalized EPSC obtained from the data presented on the left. Depolarizing iLNs during the HFS part of the PTP protocol increased potentiation $(p(-60 \mathrm{mV})=0.016, p(-40 \mathrm{mV})=0.004$, One sample two-sided $t$-test $)$. D Left, normalized EPSC before and after PTP obtained in flies in which $\mathrm{mAChR}-\mathrm{A}$ was knocked down in iLNs for holding potential of $-80 \mathrm{mV}$ (black; $n=9$ ), $-60 \mathrm{mV}$ (blue; $n=11$ ), and $-40 \mathrm{mV}$ (red; $n=9$ ). Right, mean normalized EPSC obtained from the data presented on the left. (n.s, One sample two-sided $t$-test). E. Left, normalized EPSC before and after PTP obtained in the mAChR-A-KK fly strain for holding potential of $-80 \mathrm{mV}$ (black; $n=17$ ), $-60 \mathrm{mV}$ (blue; $n=13$ ), and $-40 \mathrm{mV}$ (red; $n=16$ ). Right, mean normalized EPSC obtained from the data presented on the left. A strong potentiation was observed irrespective of the holding potential $(p(-40 \mathrm{mV})=0.000001, p(-60 \mathrm{mV})=0.0003, p(-80 \mathrm{mV})=0.00001$, One sample two-sided $t$-test). $\mathbf{F}$ Left, normalized EPSC before and after PTP obtained in the mAChR-A-KK fly strain for holding potential of $-80 \mathrm{mV}$ (black; $n=9$ ), $-60 \mathrm{mV}$ (blue; $n=12$ ), and $-40 \mathrm{mV}$ (red; $n=13$ ) in the presence of $1 \mu \mathrm{M}$ atropine. Right, mean normalized EPSC obtained from the data presented on the left. Application of atropine shifts mAChR-A-KK to the low activity state and abolishes voltage-independent potentiation (n.s, One sample two-sided $t$-test). For all panels, shaded error bands represent the SEM, lines represent single flies and GH298-GAL4 was used to drive UAS-GFP. Source data are provided as a Source Data file.

there was substantial potentiation at the more depolarized membrane potential, no potentiation was observed at the hyperpolarized membrane potential (Fig. 2C). Knocking down mAChR-A specifically in iLNs abolished the potentiation irrespective of membrane potential, confirming that PTP is controlled primarily by mAChR-A (Fig. 2D). No potentiation was observed when the iLN membrane potential was held at $-40 \mathrm{mV}$ but without applying the potentiation protocol (Supplementary Fig. 4A). These results suggest that the change in membrane potential shifts mAChR-A from the low activity state to a higher activity state and this shift in activity is required for the PTP. We therefore hypothesized that since mAChR-A-KK is "stuck" at the high activity state, it should exhibit potentiation values similar to those achieved by depolarization. Similarly, applying atropine, which maintains the voltage-independent $\mathrm{mAChR}-\mathrm{A}-\mathrm{KK}$ in the low activity state (Fig. 1C, G), should result in a weaker potentiation than $w t$ mAChR-A, or even produce no potentiation at all. In accordance with our hypothesis, applying the same protocol to flies carrying the mAChR-A-KK mutation, resulted in voltageindependent potentiation at all membrane potentials (Fig. 2E), which was not observed using a lower frequency stimulation (Supplementary Fig. 4B). In addition, atropine at a concentration that maintains the mAChR-A-KK in the low activity state (Fig. 1G), completely abolished neuronal potentiation (Fig. 2F). Taken together, these results demonstrate that $\mathrm{mAChR}-\mathrm{A}$ voltage dependence is required for efficient recruitment of mAChRA and participates in physiological plasticity processes.

A voltage-dependent $\mathrm{mAChR}-\mathrm{A}$ and nicotinic receptors crosstalk. Recently, $\mathrm{M}_{2} \mathrm{R}$ basal activity, which reflects spontaneous coupling of $\mathrm{M}_{2} \mathrm{R}$ to its cognate G-protein, was shown to also be voltage dependent ${ }^{48}$. In another study, activation of 

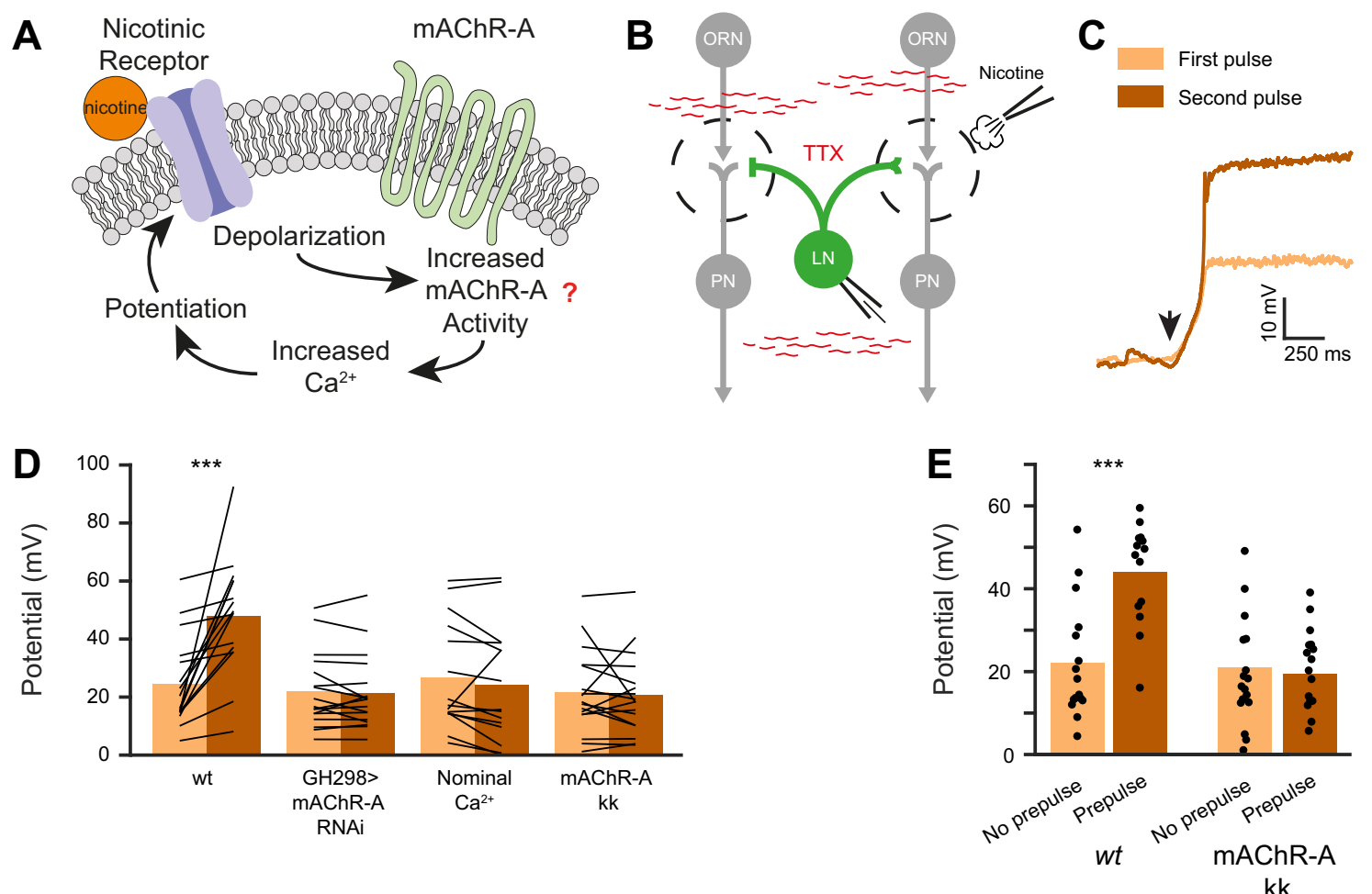

Fig. 3 mAChR-A nicotinic induced potentiation is voltage dependent. A A diagram of the suggested crosstalk between nicotinic receptors and $m A C h R-A$. Depolarization induced by activation of the nicotinic receptors can increase the constitutive activity of mAChR-A. The increased mAChR-A activity results in potentiation of the nicotinic response. B A diagram of the experimental configuration. Network activity was blocked using TTX (1 $\mu$ M), two pulses of nicotine $(100 \mu \mathrm{M})$ were injected into the AL with a temporal interval of $1 \mathrm{~min}$, and the iLN excitatory postsynaptic potential was measured using in vivo whole-cell current clamp. C Representative traces obtained from a single fly demonstrating the potentiation occurring in the second nicotinic pulse (dark brown) relative to the first pulse (light brown). D Nicotinic induced double pulse potentiation (first pulse shown in light brown, second shown in dark brown) was observed in wt flies $(n=16)$. No potentiation was observed when mAChR-A was knocked down in iLNs using GH298-GAL4 and UAS-mAChRA RNAi $(n=17)$, in nominal $\mathrm{Ca}^{2+}(n=15)$, and in the mAChR-A-KK fly strain $(n=17)$. Each line represents a single fly $(p(w t)=0.0005$, Paired sample two-sided $t$-test). E A depolarizing pre-pulse $(40 \mathrm{mV}$ for $30 \mathrm{~s}$ ) before the nicotinic pulse potentiated the response to nicotine in wt flies (No pre-pulse; $n=16$, Pre-pulse; $n=14$ ) but not in the mAChR-A-KK fly strain (No pre-pulse; $n=17$, Pre-pulse; $n=16$ ). First pulse data are the same as in panel $\mathbf{D}$. Each dot represents a single fly $p(w t)=0.00008$, Two sample two-sided $t$-test).

mAChR-A inhibited potassium currents, thereby increasing the $\mathrm{Ca}^{2+}$ level and eventually inducing the potentiation of the nicotinic receptors ${ }^{47}$. These two observations raise the possibility that depolarization induced by the activation of nicotinic receptors can increase the constitutive activity of mAChR-A and result in potentiation of the nicotinic response (Fig. 3A). We therefore examined whether two consecutive nicotine pulses would result in potentiation of the second nicotine pulse (Fig. 3B). This was indeed the case in flies expressing $w t$ mAChR-A (Fig. 3C, D). This double pulse potentiation was dependent on mAChR-A activity as it was abolished when mAChR-A was knocked down (Fig. 3D). Using nominal external $\mathrm{Ca}^{2+}$ also abolished the paired pulse potentiation, further supporting our conclusion that this potentiation involves the pathway described for mAChR-A potentiation (Fig. 3D). As there is no exogenous mAChR-A agonist, this protocol is not expected to give rise to increased potentiation in flies expressing the mAChR-A-KK mutation. Rather, since these receptors are insensitive to membrane potential, the basal activity would be expected to be unaffected by nicotine and no potentiation will be observed. Indeed, applying the paired-pulse potentiation in mAChR-A-KK flies did not elicit any potentiation to the second nicotine pulse (Fig. 3D).

These results demonstrate that activation of nicotinic receptors recruits mAChR-A which in turn potentiate the nicotinic receptors. As nicotine does not activate muscarinic receptors and since nicotine had no effect on the voltage-independent
mAChR-A-KK, the most likely explanation is that the depolarization induced by the nicotine pulse recruits mAChR-A. We therefore examined whether a depolarizing pre-pulse step prior to nicotine application would induce a similar potentiation. Indeed, a depolarizing step prior to the nicotine pulse resulted in a significant increase in the potential induced by the nicotine pulse (Fig. 3E). To verify that the potentiation following the depolarizing pre-pulse is mediated by mAChR-A, we repeated the experiment using the mAChR-A-KK fly strain. No potentiation was observed in the case of the mAChR-A-KK fly strain (Fig. 3E). We also verified that $w t$ mAChR-A activity in response to muscarine, but not that of mAChR-A-KK, is potentiated following a depolarizing pre-pulse step (Supplementary Fig. 5). Together, these results indicate that the potentiation effect is mediated by mAChR-A and not by other downstream proteins (Fig. 3E). More importantly, these results elucidate a mechanism of ionotropic receptors modulation by GPCR voltage-dependent activity.

mAChR-A voltage dependency affects odor habituation. The cumulative results thus far demonstrate that mAChR-A voltage dependence participates in iLN potentiation, albeit, to artificial stimuli. We therefore examined whether similar results could be elicited by a physiological odor stimulus. iLN activity depresses over time ${ }^{23,49,50}$ and long exposure to odors was suggested to potentiate the response of iLNs ${ }^{41,51}$ (Fig. 4A). Therefore, we 
A

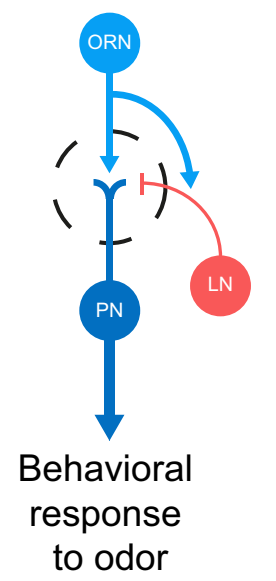

C

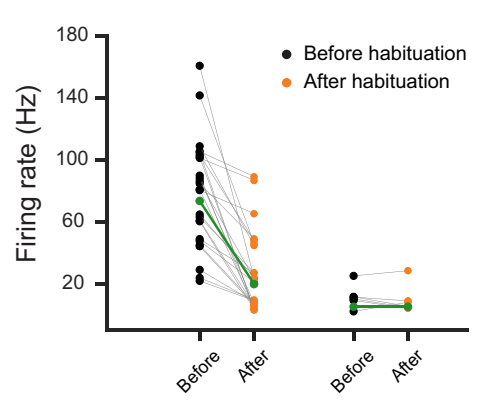

B

B wt

mAChR-A-KK

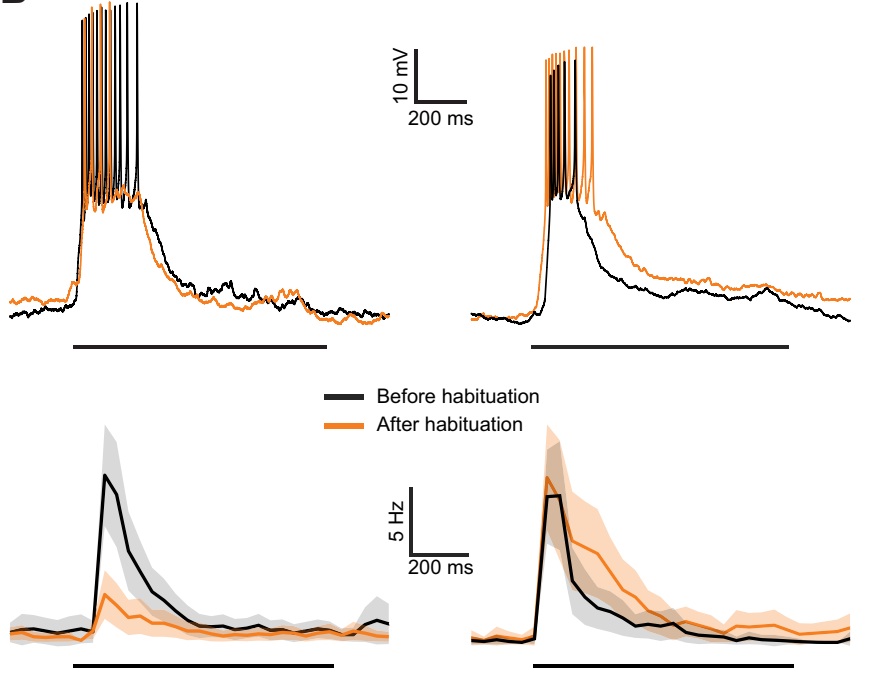

mAChR-A-KK

D

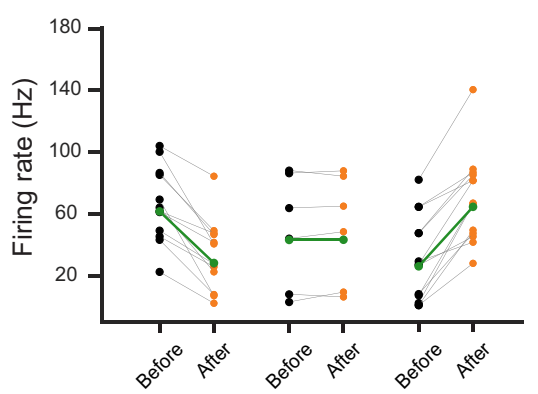

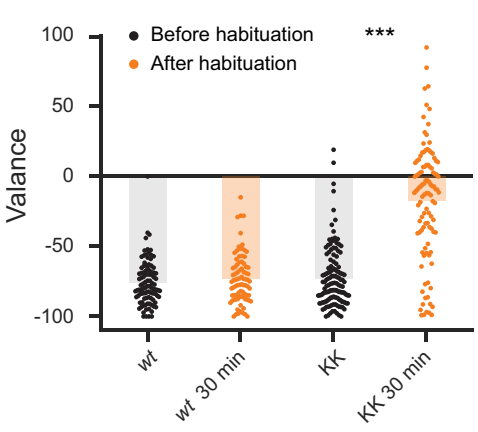

Fig. 4 mAChR-A voltage dependence affects odor habituation. A Proposed habituation mechanism: Before odor exposure, the ORN-iLN synapse is relatively weak. Thus, iLNs generate a modest inhibitory signal on the ORN-PN synapse, allowing PN activity. Following a long odor exposure, the ORN-iLN synapse is potentiated, blocking the transmission from ORNs to PNs, thereby decreasing PN activity, resulting in odor habituation. Line thickness indicates firing rate. B Top, Examples of the decrease in iLN firing rate observed in wt flies (left) and potentiation in iLN activity observed in the mAChR-A-KK fly strain (right). Black and orange denote the iLN odor response before and after odor habituation (30 min exposure) respectively. Black line indicates odor application. Bottom, iLN mean firing rate before and after odor habituation. Black and orange denote the iLN odor response before and after the odor habituation protocol respectively. ( $w t, n=31, \mathrm{mAChR-A-KK}, n=31$ ). While there is a strong decrease in iLN firing rate in $w t$ flies $(p(w t)=0.0000002$, Paired sample two-sided $t$-test), over all iLNs there is no difference in the mAChR-A-KK fly strain. Shaded error bands represent the SEM. C Maximal firing rate of the 10 stimulus trials presented to each cell, before (black) and after (orange) 30 min exposure to ethyl butyrate for data shown in panel B. Dots represent individual flies. Group averages are shown in green. In the case of wt flies $\sim 80 \%$ of flies showed a decrease in firing rate following long odor exposure. In contrast, in the mAChR-A-KK strain, only $~ 38 \%$ of flies showed a decrease firing frequency whereas $\sim 40 \%$ of flies showed potentiation. Points were randomly jittered for visualization. D The valence (negative values indicate aversion) flies assign to ethyl butyrate before and after odor habituation (30 min exposure). For wt flies, odor valance did not change, however, the mAChR-A-KK fly strain displayed a strong reduction in odor avoidance, indicating odor habituation. (wt before, $n=102$, wt after, $n=84, \mathrm{mAChR}-\mathrm{A}-\mathrm{KK}$ before, $n=139, \mathrm{mAChR}-\mathrm{A}-\mathrm{KK}$ after, $n=107, p<10^{-30}$, Two sample two sided $t$-test). Source data are provided as a Source Data file.

examined the effect of 30 min exposure to the odor ethyl butyrate on iLN activity in flies expressing $w t$ mAChR-A or mAChR-AKK. The results indicated that exposure to odor significantly decreased the firing rate in $\sim 80 \%$ of the iLNs examined in $w t$ flies (Fig. 4B, C). In contrast, the response of iLNs from mAChR-AKK flies was heterogeneous. Overall, when averaging all iLNs from $\mathrm{mAChR}-\mathrm{A}-\mathrm{KK}$ flies there was no difference in firing rate before and after odor exposure (Fig. 4B). However, a more detailed analysis revealed that in the case of mAChR-A-KK flies, long odor exposure decreased iLN firing rate in only $\sim 38 \%$ of iLNs. Contrary to $w t$ flies, there was a marked potentiation in $40 \%$ of iLNs (Fig. 4C). These results indicate potentiation of a subpopulation of iLNs in the case of the mAChR-A-KK fly strain.

These results all demonstrate that $\mathrm{mAChR}-\mathrm{A}$ voltage dependence plays a role in physiological processes and in particular in
iLN potentiation following odor exposure. We therefore examined whether these changes in potentiation also affect behavioral output. Odor habituation is a decline in behavioral response following repeated or continuous exposure to an odorant ${ }^{51}$, and has been suggested to require potentiation of iLNs ${ }^{41,52-54}$. Briefly, prior to a prolong odor exposure iLNs generate a weak inhibitory signal on the ORN-PN synapse, thus allowing the olfactory signal to efficiently propagate to higher brain regions. Following a long odor exposure, the ORN-iLN synapse is potentiated, resulting in a stronger iLN inhibitory signal, which efficiently blocks the transmission from ORNs to PNs. As a result, there is a decrease in $\mathrm{PN}$ signal to higher brain regions, resulting in odor habituation (Fig. 4A). We therefore examined whether odor habituation in mAChR-A-KK flies which have stronger iLN potentiation (Fig. 4B, C) is affected. Since mAChR-A and mAChR-A-KK 
respond similarly to a saturating level of agonist (Fig. 1D, H), one would expect that if agonist levels that reach iLNs are high enough there would be no difference in odor habituation between wt mAChR-A and mAChR-A-KK flies. However, if as expected, the agonist levels arriving to $\mathrm{mAChR}-\mathrm{A}$ on the iLNs are within the dynamic range of the dose-response curve, then one would expect to see a stronger habituation in flies with $\mathrm{mAChR}-\mathrm{A}-\mathrm{KK}$ than in wt mAChR-A flies. To test odor habituation we used custom linear chambers, each housing a single fly (Supplementary Fig. 6A). These chambers allow the presentation of an odor from either sides of the chamber while presenting odorless air flow from the other side of the chamber. The Air and odor streams converge at a central choice zone (see methods for a detailed description). Thus, each fly can choose whether to spend time in the area containing an odor. To calculate ethyl butyrate valance the odor was presented on alternating sides of the chamber for two minutes and the difference between the preference to the odor-containing side relative to the air-containing side was calculated (Supplementary Fig. 6B, see "Methods" for a detailed explanation). Following an initial odor valance measurement, flies were exposed to ethyl butyrate for $30 \mathrm{~min}$ on both sides of the chamber simultaneously and then odor valence was measured again (Supplementary Fig. 6B). The results presented in Fig. 4D and Supplementary Fig. 7A, indeed demonstrate significantly stronger habituation in $\mathrm{mAChR}-\mathrm{A}-\mathrm{KK}$ flies than in those with $w t$ mAChR-A. Since a 30-minute odor exposure did not result in strong odor habituation in $w t$ flies, we verified that habituation indeed occurs when the habituation period is prolonged to $1 \mathrm{~h}$ (Supplementary Fig. 7B). In addition, to control for a possible change in odor valance unconnected to the odor exposure, we repeated the habituation protocol without adding odor to the carrier airflow. This did not affect the odor valence in $\mathrm{mAChR}-\mathrm{A}-$ KK flies (Supplementary Fig. 7C).

We showed that iLNs undergo stronger potentiation in the mAChR-A-KK strain following odor exposure (Fig. 4B, C) and that, as one would expect from a stronger iLN potentiation, odor habituation is stronger in the mAChR-A-KK strain. However, $\mathrm{mAChR}-\mathrm{A}$ is broadly expressed in the brain ${ }^{28}$. Thus, it is possible that the effect mAChR-A-KK has on odor habituation does not arise solely from changes in iLN activity. We have previously shown that in the olfactory pathway, mAChR-A is mainly expressed and has a functional effect in iLNs of the AL and in a subset of third-order Kenyon cells (KCs) of the mushroom body $(\mathrm{MB} \text {, Fig. } 5 \mathrm{~A})^{23,28}$ which were also shown to be involved in olfactory habituation ${ }^{55}$. Therefore, we performed rescue experiments in these two cell types. To this end we used the GH298GAL4 driver which covers iLNs, and the MB247-GAL driver which covers the $\mathrm{KC}$ subtypes that express mAChR-A ${ }^{23,28}$. To rescue the effects of $\mathrm{mAChR}-\mathrm{A}-\mathrm{KK}$ we used two strategies: $\mathrm{i}$. we overexpressed a $w t$ mAChR-A using UAS-mAChR-A. ii. We knocked down mAChR-A-KK levels using UAS-mAChR-A $\mathrm{RNAi}^{23,28}$. Overexpression of a $w t$ mAChR-A in iLNs should abolish the increased habituation effect in mAChR-A-KK flies since the overexpression of a voltage-dependent $\mathrm{mAChR}-\mathrm{A}$ has a dominant effect. Similarly, knocking down the voltageindependent mAChR-A-KK should abolish its effects. We hypothesize that if the behavioral effect arising from $\mathrm{mAChR}$ $\mathrm{A}-\mathrm{KK}$ is localized to iLNs, overexpression of a $w t$ mAChR-A or knockdown of mAChR-A-KK in KCs of the MB should not change mAChR-A-KK flies' behavior. As expected, applying both strategies to iLNs resulted in no odor habituation as was seen in $w t$ flies (Fig. 5B, D), whereas, rescue attempts in KCs had no effect whatsoever on odor habituation and strong odor habituation was as observed as in the mAChR-A-KK fly strain. Together, these results support the notion that mAChR-A-KK induced potentiation of the ORN-iLN synapse underlies the increased odor habituation observed. Taken together, our results unambiguously demonstrate that the voltage dependence of $\mathrm{mAChR}-\mathrm{A}$ is crucial for both physiological process and behavioral output.

\section{Discussion}

Here we show that the Drosophila mAChR-A is voltage dependent. In addition, our results reveal that a voltage-independent receptor variant, mAChR-A-KK, exhibits altered neuronal potentiation to both artificial and physiological stimuli, and that these changes in potentiation influence behavior. Furthermore, generating conditions such that mAChR-A becomes voltage independent and resides in the low activity state, completely abolishes the mAChR-A dependent potentiation. Thus, this work represents a demonstration that voltage dependence is crucial for the normal function of GPCRs in vivo, thereby changing our understanding of GPCR recruitment and function.

GPCRs voltage dependence was discovered almost 20 years ago $^{10}$. Since then it was demonstrated for various GPCRs ${ }^{7-15,56}$. These studies provided important information on the identity of voltage-dependent GPCRs as well as on some of the mechanisms underlying GPCR voltage dependency. However, as they were performed in cell culture, whether this GPCR voltage dependency plays any physiological role was not addressed. GPRC voltage dependence was shown to control synaptic release initiation and duration in vitro ${ }^{17-22}$, and recently it was shown that membrane depolarization recruits voltage-dependent purinergic receptors in sympathetic chromaffin cells to increase the quantal size ${ }^{18}$. Nevertheless, even in these cases, there is no evidence that these small changes in the duration or strength of synaptic release affect neuronal computation or behavioral output, especially on the background of noisy neural activity. Our study, which generated a fly strain with a voltage independent muscarinic receptor allowed us to address this question and unequivocally demonstrate that GPCR voltage dependence affects neuronal computation and behavioral output. Furthermore, contrary to all previous studies which showed effects only on synaptic release, this study demonstrates that GPCR voltage dependence plays a role postsynaptically in the canonical GPCR pathway.

Since $\mathrm{mAChR}-\mathrm{A}$ voltage dependence shifts the dynamic range of the dose-response curve, it can only be relevant if $\mathrm{mAChR}-\mathrm{A}$ is exposed to sub-saturation concentrations of neurotransmitter. In this context, mammalian glutamatergic receptors are usually far from saturation during quantal transmission ${ }^{57}$. Notably, the rapid removal of neurotransmitter from the synaptic cleft also generates sub-saturation conditions ${ }^{58}$. Our results further support this notion since mAChR-A-KK has similar activity to mAChR-A at saturation levels of the receptor (Fig. 1), and differs only in the dynamic range of responses. The findings of a strong physiological effect in response to synaptic release as well as a strong behavioral effect all point to a sub-saturating agonist concentration.

The demonstration that GPCR voltage dependence has physiological implications, suggests the presence of a strong crosstalk between the ionotropic pathways (that rapidly affect membrane potential) and the metabotropic pathways. For GPCRs that exhibit increased activity upon depolarization, this crosstalk is reminiscent of the crosstalk between the glutamatergic a-amino3-hydroxy-5-methyl-4-isoxazolepropionic acid receptor (AMPAR) and N-methyl-D-aspartate receptor (NMDAR). NMDAR activity requires depolarization, which usually originates from AMPAR activation, and the recruitment of NMDAR can result in AMPA potentiation ${ }^{59}$. Although the metabotropic receptors do not require depolarization for their activity, depolarization favors their recruitment. For nicotinic and muscarinic receptors, a model arises where depolarization caused by 
A

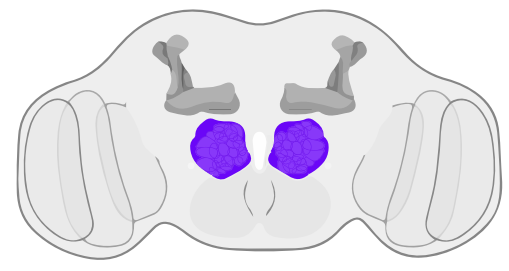

B
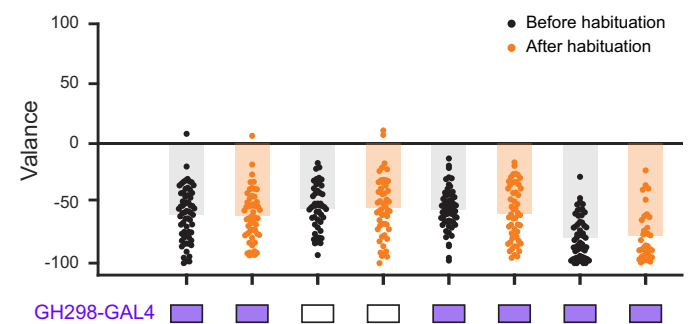

UAS-mAChR-A $\square \square \square \square \square \square \square \square \square$ mAChR-A-KK

$\mathrm{n}=$

D

$\begin{array}{llllllll}63 & 53 & 49 & 50 & 58 & 52 & 57 & 53\end{array}$

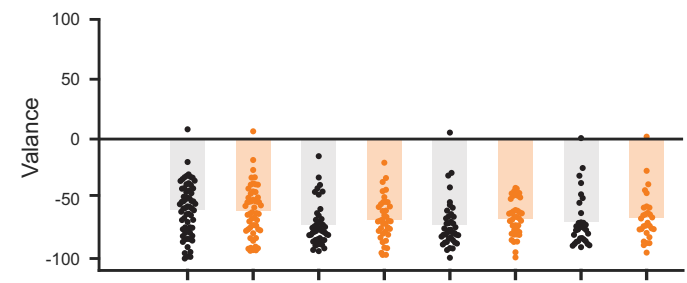

GH298-GAL4 $\square \square \square \square \square \square \square$ UAS-mAChR-A RNAi

mAChR-A-KK

$\mathrm{n}=$
iLN mAChR-A RNAi

$\begin{array}{lllll}\square & \square & \square & \square & \square \\ \square & \square & \square & \square & \square \\ \square & \square & \square & \square & \square \\ 38 & 40 & 38 & 32 & 29\end{array}$

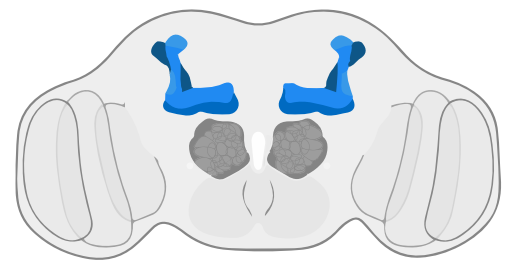

C

MB mAChR-A overexpression $\quad * * *$

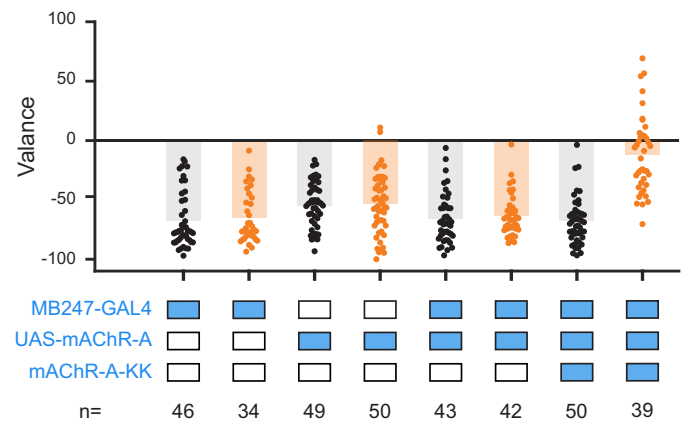

E

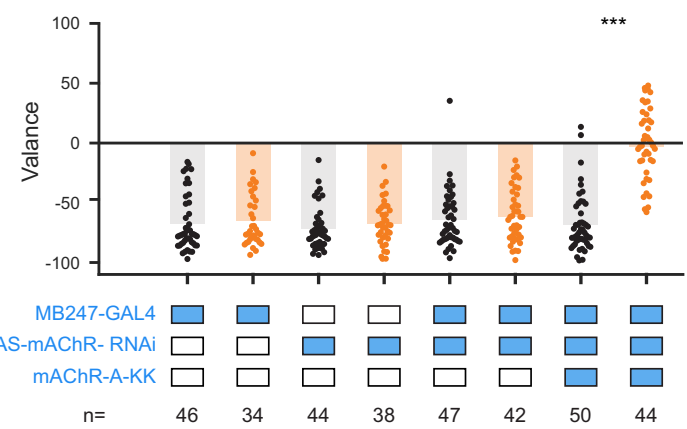

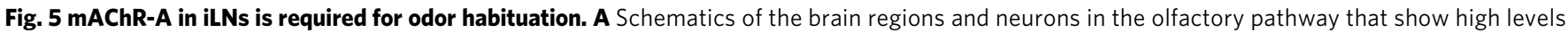

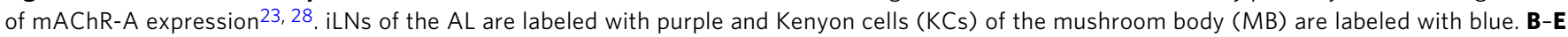
The valence (negative values indicate aversion) flies assign to ethyl butyrate before and after the odor habituation protocol (30 min exposure) were examined when the effect of the mAChR-A-KK mutation was abolished in iLNs (B, D, using the GH298-GAL driver line) or in KCs (C, E, using the MB247-GAL4 driver line). To abolish the effect of the mAChR-A-KK mutation we used either overexpression of wt mAChR-A (B, C) or knockdown of the mAChR-A-KK using RNAi (D, E). While reducing mAChR-A-KK in iLNs using both methods abolished the observed strong odor habituation, similar manipulation in KCs of the MB had no effect and flies still showed strong odor habituation $\left(n=33-64, p(p a n e l \mathbf{C})<10^{-10}\right.$, $\mathrm{p}(\mathrm{panel} \mathbf{E})<10^{-10}$, Two-sample two sided t-test). GH298-GAL4 data are the same for panels B and D, MB247-GAL4 data are the same for panels C and D. Source data are provided as a Source Data file.

activation of nicotinic receptors strongly affects the activity of the co-activated muscarinic receptor. In turn, the improved recruitment of the muscarinic receptors results in potentiation of the nicotinic receptors (Fig. 3A). The above suggestion is not limited only to the muscarinic receptors but can also be extended to other GPCRs. For example, we have recently demonstrated a strong similarity between the function of mAChR-A in iLNs and the function of the glutamatergic mGluR1 expressed in inhibitory granule cells of the mammalian olfactory bulb ${ }^{23,60}$. It is interesting to note that this similarity is further extended to their voltage dependence and mGluR1 shifts to a high activity state following depolarization ${ }^{14}$. It is thus possible that similar to the Drosophila muscarinic receptor, mGluR1 voltage dependency participates in a crosstalk between glutamatergic ionotropic and metabotropic receptors and has a role in mammalian olfactory processing.

In Drosophila, odor habituation is thought to be mediated by the potentiation of iLNs ${ }^{41,51}$. Our results (Figs. 4 and 5) are in agreement with the role of iLNs in odor habituation and demonstrate that cholinergic neuromodulation by mAChR-A is an important step in odor habituation. Our results further show that $\mathrm{mAChR}-\mathrm{A}$ voltage dependence plays a role in odor habituation (Figs. 4 and 5). It is interesting to note that odor habituation requires $\mathrm{PN}$ input onto iLNs ${ }^{54}$. The requirement of $\mathrm{PN}$ input can explain how odor habituation occurs in a glomerulusspecific manner (i.e. according to the identity of the activated $\mathrm{PNs})^{51}$. However, PNs generally respond in a non-linear manner to different concentrations, with relatively low odor concentrations required to saturate PN activity ${ }^{61-63}$. Thus, even low odor concentrations should generate strong odor habituation, contrary to observed results ${ }^{55}$. Our results, which show that mAChR-A voltage dependence plays a role in odor habituation (Figs. 4 and 5), may resolve this discrepancy. Contrary to PNs, iLNs are linearly recruited by $\mathrm{ORNs}^{39}$. Thus at low odor concentration iLNs will undergo only weak depolarization. Therefore, although the relevant PN will already be saturated, due to the low iLN depolarization, the responding $\mathrm{mAChR}-\mathrm{A}$ will be in the low activity state and the overall habituation will not be strong. In contrast, at 
a high odor concentration, iLNs will undergo strong depolarization, shifting mAChR-A to the high activity state, and as a result habituation will be stronger. In this way, GPCR voltage dependence can act as a rheostat that allows for a gradual increase in neuromodulation.

Taken together, this work provides a demonstration of a physiological role for the voltage dependency of GPCRs and may serve as a paradigm shift in our understanding of neural function and drug discovery.

\section{Methods}

Fly strains. Fly strains (see below) were raised on cornmeal agar under a $12 \mathrm{~h}$ light/ $12 \mathrm{~h}$ dark cycle at $25^{\circ} \mathrm{C}$. The following transgenes were used: GH298-GAL4 (Bloomington \#37294), MB247-GAL4 ${ }^{64}$, UAS-mAChR-A RNAi (TRiP.JF02725, Bloomington \#27571), UAS-mCD8-GFP (Bloomington \#32185), UAS-GCaMP6f (Bloomington \#42747) and w[1118] (Bloomington \#32185 5905).

The mAChR-A-KK fly strain was generated by GenetiVision (see supplemental methods). UAS-mAChR-A was inserted into the Drosophila pBID-UASC plasmid and flies were generated by BestGene Inc.

Oocytes. Xenopus laevis oocytes were isolated and incubated in NDE96 solution composed of ND96 (96 mM NaCl, $2 \mathrm{mM} \mathrm{KCl}, 1 \mathrm{mM} \mathrm{CaCl}, 1 \mathrm{mM} \mathrm{MgCl}$, and $5 \mathrm{mM}$ Hepes, $\mathrm{pH}$ adjusted to 7.5 with $\mathrm{NaOH}$ ), with the addition of $2.5 \mathrm{mM} \mathrm{Na}{ }^{+}$ pyruvate, $100 \mathrm{U} / \mathrm{ml}$ penicillin and $100 \mathrm{mg} / \mathrm{ml}$ streptomycin ${ }^{65}$. One day after their isolation, the oocytes were injected with the following cRNAs: mAChR-A and its mutations (1 ng/oocyte) GIRK1 and GIRK2 (200 pg/oocyte for each) and Gai3 ( $2 \mathrm{ng} /$ oocyte). For experiments designed to measure $\beta$-arrestin activation cRNAs of GRK3 (1 ng/oocyte) and $\beta$-arrestin 2 ( $5 \mathrm{ng} /$ oocyte) were co-injected. Gq activated $\mathrm{Ca}^{2+}$ dependent $\mathrm{Cl}^{-}$currents were measured in oocytes injected with $5 \mathrm{ng} /$ oocyte of the mAChR-A without the co-injection of other cRNAs.

cDNA plasmids were linearized with the appropriate restriction enzymes. Point mutations were prepared using Quick-Change II Site-Directed Mutagenesis Kit (Stratagene, La Jolla,CA, USA).

All experimental procedures used in this study were performed in accordance with relevant guidelines and regulations, and were approved by the Hebrew University's Animal Care and Use Committee (Ethical approval number NS-1112909-3).

The currents were recorded 3-5 days after cRNA injection by using two electrode voltage clamp amplifier ${ }^{10}$ (Warner OC $725 \mathrm{C}$ amplifier, Warner Instruments, Hamden, CT). An oocyte was placed in the recording bath containing ND96 solution and was impaled by two electrodes pulled from $1.5 \mathrm{~mm}$ borosilicate capillaries (Warner instruments). Both electrodes were filled with $3 \mathrm{M} \mathrm{KCl}$ solution. The electrode resistance was between 0.5 and $2 \mathrm{M} \Omega$. mAChR-A mediated GIRK currents were measured in a solution of $24 \mathrm{mM} \mathrm{K}^{+}, 72 \mathrm{mM} \mathrm{NaCl}, 24 \mathrm{mM}$ $\mathrm{KCl}, 1 \mathrm{mM} \mathrm{CaCl}_{2}, 1 \mathrm{mM} \mathrm{MgCl}_{2}$, and $5 \mathrm{mM}$ Hepes, $\mathrm{pH}$ adjusted to 7.5 with $\mathrm{KOH}$. mAChR-A mediated $\mathrm{Ca}^{2+}$ dependent $\mathrm{Cl}^{-}$currents were measured in ND96 solution. pCLAMP10 software (Axon Instruments) was used for data acquisition.

The dose-response curves were fitted by the following equation: $Y=$ Bottom + $\mathrm{X}^{*}$ (Top-Bottom)/ $(\mathrm{EC} 50+\mathrm{X})$, where $\mathrm{Y}$ is the normalized response, $\mathrm{X}$ is the concentration of $\mathrm{ACh}$, and EC50 is the ACh concentration that gives the half maximal response. $\beta$-arrestin mediated desensitization was calculated as the percentage of GIRK current that remains after $80 \mathrm{sec}$ continuous ACh application from the peak GIRK current.

\section{Olfactory stimulation. Ethyl butyrate was purchased from Sigma-Aldrich} (Rehovot, Israel) and was at the purest level available. ACV was bought at a local supermarket (RAUCH Fruchtsäfte GmbH \& Co OG apple cider vinegar). Odors at $10^{-1}$ dilution were delivered by switching mass-flow controlled carrier at $0.4 \mathrm{l} / \mathrm{min}$ and stimulus stream (ms at $0.4 \mathrm{l} / \mathrm{min}$ (Sensirion) via software-controlled solenoid valves (The Lee Company). This resulted in a final dilution of $5 \times 10^{-2}$ of odor delivered to the fly. Air-streamed odor was delivered through a 1/16 inch ultrachemical-resistant Versilon PVC tubing (Saint-Gobain, NJ, USA) that was placed $5 \mathrm{~mm}$ from the fly's antenna.

Electrophysiology. Flies were anesthetized on ice, then a single fly was moved to a custom-built chamber and fixed to aluminum foil using wax. The cuticle and trachea in the required area were removed, and the exposed brain was superfused with carbonated solution $\left(95 \% \mathrm{O}_{2}, 5 \% \mathrm{CO}_{2}\right)$ containing $103 \mathrm{mM} \mathrm{NaCl}, 3 \mathrm{mM} \mathrm{KCl}$, $5 \mathrm{mM}$ trehalose, $10 \mathrm{mM}$ glucose, $26 \mathrm{mM} \mathrm{NaHCO} 3,1 \mathrm{mM} \mathrm{NaH} \mathrm{PO}_{4}, 1.5 \mathrm{mM}$ $\mathrm{CaCl}_{2}, 4 \mathrm{mM} \mathrm{MgCl}_{2}$, and $5 \mathrm{mM} \mathrm{N}$-Tris (TES), $\mathrm{pH}$ 7.3. Nominal external $\mathrm{Ca}^{2+}$ was the same except that no $\mathrm{Ca}^{2+}$ was added. Sample sizes were based on previous reports in the field. In vivo whole-cell current clamp recordings were made as previously described ${ }^{66}$ on 2-4 day old male and female flies. Briefly, a driver line was used to drive GFP in target neurons and the fly brains were visualized with a Scientifica SliceScope Pro 1000 upright microscope with a 40x water immersion objective. Patch pipettes with a resistance of 9-12 $\mathrm{M} \Omega$ were filled with a solution containing: $140 \mathrm{mM}$ potassium aspartate, $10 \mathrm{mM}$ Hepes, $1 \mathrm{mM} \mathrm{KCL}, 4 \mathrm{mM}$ MgATP, $0.5 \mathrm{mM} \mathrm{Na}_{3} \mathrm{GTP}$, and $1 \mathrm{mM}$ EGTA. The $\mathrm{pH}$ of the solution was adjusted to 7.3 and osmolarity to $265 \mathrm{mOsm}$. Voltage was acquired using an Axon Instruments MultiClamp 700B in current-clamp mode, then was digitized at $50 \mathrm{kHz}$, and low-pass filtered at $1 \mathrm{kHz}$. A small constant hyperpolarizing current was applied to maintain a membrane potential of $-60 \mathrm{mV}$ upon achieving a gigaseal and during the subsequent break-in.

Functional imaging. Functional Imaging was performed as previously described $^{28,67,68}$ using a two-photon laser-scanning microscopy (DF-Scope installed on an Olympus BX51WI microscope). Flies were prepared as described for the electrophysiology experiments. Fluorescence was excited by a Ti-Sapphire laser (Mai Tai HP DS, $100 \mathrm{fs}$ pulses) centered at $910 \mathrm{~nm}$, attenuated by a Pockels cell (Conoptics) and coupled to a galvo-resonant scanner. Excitation light was focused by a 20X, 1.0 NA objective (Olympus XLUMPLFLN20XW), and emitted photons were detected by GaAsP photomultiplier tubes (Hamamatsu Photonics, H10770PA-40SEL), whose currents were amplified (Hamamatsu HC-130-INV) and transferred to the imaging computer (MScan 2.3.01). All imaging experiments were acquired at $30 \mathrm{~Hz}$.

High-frequency stimulation application. Antennal nerve stimulation was as described previously ${ }^{63}$. Briefly, a glass pipette was pulled and broken to create a narrow opening that allowed the antennal nerve to be sucked in. A constant current stimulator (Digitimer, DS3 Isolated Current Stimulator) was controlled by custom LabView script. Shock intensity was selected on the basis of the minimal stimulus needed to generate an excitatory postsynaptic current that was stable for the baseline period. All stimuli were in the range of 150-230 mA, and each lasted $50 \mu \mathrm{s}$. The high-frequency stimulation (HFS) protocol was as follows: 5 stimuli at $0.016 \mathrm{~Hz}$ were used as a baseline followed by HFS at $100 \mathrm{~Hz}$ for $1 \mathrm{sec}$ at the selected holding potential, followed by 10 stimuli at $0.016 \mathrm{~Hz}$. The peaks of the excitatory postsynaptic current (EPSC) were extracted using custom MATLAB scripts. In cases when the electrical stimulus elicited an action potential, the extracted value was the current at which the action potential was initiated as indicated by a sharp change in the current derivative.

Pharmacology. The following drugs were used: atropine (Sigma-Aldrich \#A0132), nicotine (Sigma-Aldrich \#N3876), muscarine (Sigma-Aldrich \#M6532), acetylcholine (Sigma-Aldrich \#A6625) and TTX (Alomone Labs \#T-550). In all cases, stock solutions were prepared were diluted to the final concentration before experiments. Drugs were applied either by bath application or were injected directly to the AL using a pico-injector (Harvard Apparatus, PLI-100).

Behavioral experiment. For behavior experiments, 7-10 days old male and female fed flies were used. Experiments were performed in a custom-built, fully automated apparatus as previously described $28,69,70$. Briefly, single flies were placed in clear polycarbonate chambers (length $50 \mathrm{~mm}$, width $5 \mathrm{~mm}$, height $1.3 \mathrm{~mm}$ ). For the ACV experiment, starved flies were used. Starved flies were placed in a vial containing water-soaked filter paper $24 \mathrm{~h}$ prior to the experiment. All flies were backcrossed except for $w t$ and mAChR-A-KK strains that cannot be backcrossed. All experiments were performed during the flies' active hours. Air or odor streams were presented from each side and converged at a central choice zone. Mass flow controllers (CMOSens PerformanceLine, Sensirion) were used to control air flow. A carrier flow $(2.7 \mathrm{l} / \mathrm{min})$ was combined with an odor stream $(0.3 \mathrm{l} / \mathrm{min})$ obtained by circulating the air flow through vials filled with a liquid odorant resulting in a $3 \mathrm{l} / \mathrm{min}$ total flow that was split between the 20 chambers. As a result, the total flow in each half chamber was $0.15 \mathrm{l} / \mathrm{min}$. Two identical odor delivery systems delivered odors independently to each half of the chamber. Ethyl butyrate and ACV were prepared daily at 10 fold dilution in mineral oil or water respectively which was further 10 fold diluted with the carrier air stream to yield a final 100 fold odor dilution.

The 20 chambers were stacked in two columns each containing 10 chambers and were backlit by $940 \mathrm{~nm}$ LEDs (Vishay TSAL6400). Images were obtained by a MAKO CMOS camera (Allied Vision Technologies) equipped with a Computer M0814-MP2 lens. The apparatus was operated in a temperature controlled incubator (Panasonic MIR 154) at $25^{\circ} \mathrm{C}$. A virtual instrument written in LabVIEW 7.1 (National Instruments) extracted fly position data from video images and controlled the delivery of odors. Data were analyzed in MATLAB 2018a (The MathWorks).

For odor habituation experiments, an odor valence protocol was performed which was composed of odor from the left side and airflow from the right side of the chamber for $2 \mathrm{~min}$ followed by $2 \mathrm{~min}$ of opposite sides (i.e. odor from the right side of the chamber). This was followed by an odor habituation protocol in which the odor was presented from both sides of the chamber for 30 min or $1 \mathrm{~h}$. After the habituation period the odor valence protocol was repeated. The valance index was calculated as (preference for the left side when it contains odor) - (preference for the left side when it contains air).

Quantification and statistical analysis. All statistical testing and parameter extraction were done using custom MATLAB code (The MathWorks, Inc.), Prism 
6 (GraphPad), or SPSS (IBM Corp.). Significance was defined as a p-value smaller than 0.05 and all statistical tests were two-sided. For all figures, error bars and shaded areas represent the standard error of the mean (SEM).

For presentation, bar plots with dots were generated using the UnivarScatter MATLAB ToolBox (https://www.mathworks.com/matlabcentral/fileexchange/ 54243-univarscatter) and the shadedErrorBar function (https://github.com/ raacampbell/shadedErrorBar) for shaded errors on traces.

Reporting summary. Further information on research design is available in the Nature Research Reporting Summary linked to this article.

\section{Data availability}

Source data are provided with this paper. The data used to generate the figures are available in the following GitHub page: https://github.com/ParnasLab. Source data are provided with this paper.

\section{Code availability}

The code used to generate the figures is available in the following GitHub page: https:// github.com/ParnasLab.

Received: 27 June 2021; Accepted: 29 November 2021; Published online: 13 December 2021

\section{References}

1. Hanlon, C. D. \& Andrew, D. J. Outside-in signaling - a brief review of GPCR signaling with a focus on the Drosophila GPCR family. J. Cell Sci. 128, 3533-3542 (2015).

2. Huang, Y. \& Thathiah, A. Regulation of neuronal communication by G protein-coupled receptors. FEBS Lett. 589, 1607-1619 (2015).

3. Dannhäuser, S. et al. Antinociceptive modulation by the adhesion GPCR CIRL promotes mechanosensory signal discrimination. Elife 9, 1-43 (2020).

4. Ahmad, R. \& Dalziel, J. E. G protein-coupled receptors in taste physiology and pharmacology. Front. Pharmacol. 11, 1771 (2020).

5. Rosenbaum, D. M., Rasmussen, S. G. F. \& Kobilka, B. K. The structure and function of G-protein-coupled receptors. Nature 459, 356-363 (2009).

6. Venkatakrishnan, A. J. et al. Molecular signatures of G-protein-coupled receptors. Nature 494, 185-194 (2013).

7. Ågren, R. \& Sahlholm, K. Voltage-dependent dopamine potency at D1-like dopamine receptors. Front. Pharmacol. 11, 581151 (2020).

8. Kurz, M., Krett, A. L. \& Bünemann, M. Voltage dependence of prostanoid receptors. Mol. Pharmacol. 97, 267-277 (2020).

9. Ruland, J. G., Kirchhofer, S. B., Klindert, S., Bailey, C. P. \& Bünemann, M. Voltage modulates the effect of $\mu$-receptor activation in a ligand-dependent manner. Br. J. Pharmacol. 177, 3489-3504 (2020).

10. Ben-Chaim, Y., Tour, O., Dascal, N., Parnas, I. \& Parnas, H. The M2 muscarinic G-protein-coupled receptor is voltage-sensitive. J. Biol. Chem. 278, 22482-22491 (2003).

11. Ben-Chaim, Y. et al. Movement of 'gating charge' is coupled to ligand binding in a G-protein-coupled receptor. Nature 444, 106-109 (2006).

12. Rinne, A., Birk, A. \& Bünemann, M. Voltage regulates adrenergic receptor function. Proc. Natl Acad. Sci. U. S. A. 110, 1536-1541 (2013).

13. Gurung, I. S., Martinez-Pinna, J. \& Mahaut-Smith, M. P. Novel consequences of voltage-dependence to G-protein-coupled P2Y1 receptors. Br. J. Pharmacol. 154, 882-889 (2009).

14. Ohana, L., Barchad, O., Parnas, I. \& Parnas, H. The metabotropic glutamate G-protein-coupled receptors mGluR3 and mGluR1a are voltage-sensitive. J. Biol. Chem. 281, 24204-24215 (2006).

15. Sahlholm, K., Nilsson, J., Marcellino, D., Fuxe, K. \& Århem, P. Voltagedependence of the human dopamine D2 receptor. Synapse 62, 476-480 (2008).

16. Bezanilla, F. How membrane proteins sense voltage. Nat. Rev. Mol. Cell Biol. 9, 323-332 (2008).

17. Parnas, H. \& Parnas, I. The chemical synapse goes electric: $\mathrm{Ca}^{2+}$ - and voltagesensitive GPCRs control neurotransmitter release. Trends Neurosci. 30, 54-61 (2007).

18. Zhang, Q. et al. Regulating quantal size of neurotransmitter release through a GPCR voltage sensor. Proc. Natl Acad. Sci. USA 117, 26985-26995 (2020).

19. Kupchik, Y. M. et al. A novel fast mechanism for GPCR-mediated signal transduction-control of neurotransmitter release. J. Cell Biol. 192, 137-151 (2011).

20. Khanin, R., Parnas, I. \& Parnas, H. On the feedback between theory and experiment in elucidating the molecular mechanisms underlying neurotransmitter release. Bull. Math. Biol. 68, 997-1009 (2006).
21. Ilouz, N., Branski, L., Parnis, J., Parnas, H. \& Linial, M. Depolarization affects the binding properties of muscarinic acetylcholine receptors and their interaction with proteins of the exocytic apparatus. J. Biol. Chem. 274, 29519-29528 (1999).

22. Kupchik, Y. M. et al. Molecular mechanisms that control initiation and termination of physiological depolarization-evoked transmitter release. Proc. Natl Acad. Sci. USA 105, 4435-4440 (2008).

23. Rozenfeld, E., Lerner, H. \& Parnas, M. Muscarinic modulation of antennal lobe GABAergic local neurons shapes odor coding and behavior. Cell Rep. 29, 3253-3265 (2019)

24. Ren, G. R., Folke, J., Hauser, F., Li, S. \& Grimmelikhuijzen, C. J. P. The A- and B-type muscarinic acetylcholine receptors from Drosophila melanogaster couple to different second messenger pathways. Biochem. Biophys. Res. Commun. 462, 358-364 (2015).

25. Collin, C. et al. Two types of muscarinic acetylcholine receptors in Drosophila and other arthropods. Cell. Mol. Life Sci. 70, 3231-3242 (2013).

26. Croset, V., Treiber, C. D. \& Waddell, S. Cellular diversity in the Drosophila midbrain revealed by single-cell transcriptomics. Elife https://doi.org/10.7554/ elife.34550 (2018)

27. Davie, K. et al. A single-cell transcriptome atlas of the aging Drosophila brain. Cell https://doi.org/10.1016/j.cell.2018.05.057 (2018).

28. Bielopolski, N. et al. Inhibitory muscarinic acetylcholine receptors enhance aversive olfactory learning in adult Drosophila. Elife 8, e48264 (2019).

29. Buck, L. \& Axel, R. A novel multigene family may encode odorant receptors: a molecular basis for odor recognition. Cell 65, 175-187 (1991).

30. Vosshall, L. B., Amrein, H., Morozov, P. S., Rzhetsky, A. \& Axel, R. A spatial map of olfactory receptor expression in the Drosophila antenna. Cell 96, 725-736 (1999).

31. Gao, Q., Yuan, B. \& Chess, A. Convergent projections of Drosophila olfactory neurons to specific glomeruli in the antennal lobe. Nat. Neurosci. 3, 780-785 (2000).

32. Mombaerts, P. et al. Visualizing an olfactory sensory map. Cell 87, 675-686 (1996).

33. Vosshall, L. B., Wong, A. M. \& Axel, R. An olfactory sensory map in the fly brain. Cell 102, 147-159 (2000).

34. Tanaka, N. K., Endo, K. \& Ito, K. Organization of antennal lobe-associated neurons in adult Drosophila melanogaster brain. J. Comp. Neurol. 520, 4067-4130 (2012).

35. Liu, W. W. \& Wilson, R. I. Glutamate is an inhibitory neurotransmitter in the Drosophila olfactory system. Proc. Natl Acad. Sci. USA 110, 10294-10299 (2013).

36. Root, C. M. et al. A presynaptic gain control mechanism fine-tunes olfactory behavior. Neuron 59, 311-321 (2008).

37. Galizia, C. G. Olfactory coding in the insect brain: data and conjectures. Eur. J. Neurosci. 39, 1784-1795 (2014).

38. Olsen, S. R. \& Wilson, R. I. Lateral presynaptic inhibition mediates gain control in an olfactory circuit. Nature 452, 956-960 (2008).

39. Olsen, S. R., Bhandawat, V. \& Wilson, R. I. Divisive normalization in olfactory population codes. Neuron 66, 287-299 (2010).

40. Mohamed, A. A. M. et al. Odor mixtures of opposing valence unveil interglomerular crosstalk in the Drosophila antennal lobe. Nat. Commun. https:// doi.org/10.1038/s41467-019-09069-1 (2019).

41. Das, S. et al. Plasticity of local GABAergic interneurons drives olfactory habituation. Proc. Natl. Acad. Sci. https://doi.org/10.1073/pnas.1106411108 (2011)

42. Osman, S. et al. Plasticity of recurrent inhibition in the Drosophila antennal lobe. J. Neurosi. https://doi.org/10.1523/jneurosci.1099-12.2012 (2012).

43. Masse, N. Y., Turner, G. C. \& Jefferis, G. S. X. E. Olfactory Information Processing in Drosophila. Current Biology https://doi.org/10.1016/ j.cub.2009.06.026 (2009)

44. Barchad-Avitzur, O. et al. A novel voltage sensor in the orthosteric binding site of the M2 muscarinic receptor. Biophys. J. 111, 1396-1408 (2016).

45. Wess, J. G-protein-coupled receptors: molecular mechanisms involved in receptor activation and selectivity of G-protein recognition. FASEB J. 11, 346-354 (1997).

46. Wess, J., Bonner, T. I., Dörje, F. \& Brann, M. R. Delineation of muscarinic receptor domains conferring selectivity of coupling to guanine nucleotidebinding proteins and second messengers. Mol. Pharmacol. 38, 517-523 (1990).

47. Moreau, E. et al. Orthosteric muscarinic receptor activation by the insect repellent IR3535 opens new prospects in insecticide-based vector control. Sci. Rep. 10, 1-15 (2020).

48. Ben-Chaim, Y., Broide, C. \& Parnas, H. The coupling of the M2 muscarinic receptor to its G protein is voltage dependent. PLoS One 14, e0224367 (2019).

49. Martelli, C. \& Fiala, A. Slow presynaptic mechanisms that mediate adaptation in the olfactory pathway of Drosophila. Elife 8 (2019).

50. Nagel, K. I. \& Wilson, R. I. Mechanisms Underlying Population Response Dynamics in Inhibitory Interneurons of the Drosophila Antennal Lobe. J. Neurosci. https://doi.org/10.1523/jneurosci.3887-15.2016 (2016).

51. Twick, I., Lee, J. A. \& Ramaswami, M. Olfactory habituation in Drosophilaodor encoding and its plasticity in the antennal lobe. In Progress in Brain 
Research. Odor Memory and Perception (eds Barkai, E. \& Wilson, D. A.). vol. 208 3-38 (Elsevier B.V., 2014).

52. Larkin, A. et al. Central synaptic mechanisms underlie short-term olfactory habituation in Drosophila larvae. Learn. Mem. 17, 645-653 (2010).

53. Sadanandappa, M. K. et al. Synapsin function in GABA-ergic interneurons is required for short-term olfactory habituation. J. Neurosci. 33, 16576-16585 (2013).

54. Sudhakaran, I. P. et al. Plasticity of recurrent inhibition in the Drosophila antennal lobe. J. Neurosci. 32, 7225-7231 (2012).

55. Semelidou, O., Acevedo, S. F. \& Skoulakis, E. M. C. Temporally specific engagement of distinct neuronal circuits regulating olfactory habituation in Drosophila. Elife 7, e39569 (2018).

56. Stanfield, P. Voltage sparks a GPCR. Nat. Cell Biol. 8, 1323-1325 (2006).

57. Liu, G., Choi, S. \& Tsien, R. W. Variability of neurotransmitter concentration and nonsaturation of postsynaptic AMPA receptors at synapses in hippocampal cultures and slices. Neuron 22, 395-409 (1999).

58. Barberis, A., Petrini, E. M. \& Mozrzymas, J. W. Impact of synaptic neurotransmitter concentration time course on the kinetics and pharmacological modulation of inhibitory synaptic currents. Front. Cellular Neurosci. 5, 6 (2011).

59. Kandel, E. R. et al. Principles of Neural Science (The McGraw-Hill Companies Inc., 2013).

60. Heinbockel, T., Laaris, N. \& Ennis, M. Metabotropic Glutamate Receptors in the Main Olfactory Bulb Drive Granule Cell-Mediated Inhibition. I. Neurophysiol. https://doi.org/10.1152/jn.00884.2006. (2006)

61. Stopfer, M., Jayaraman, V. \& Laurent, G. Intensity versus identity coding in an olfactory system. Neuron 39, 991-1004 (2003).

62. Bhandawat, V., Olsen, S. R., Gouwens, N. W., Schlief, M. L. \& Wilson, R. I. Sensory processing in the Drosophila antennal lobe increases reliability and separability of ensemble odor representations. Nat. Neurosci. 10, 1474-1482 (2007).

63. Kazama, H. \& Wilson, R. I. Homeostatic matching and nonlinear amplification at identified central synapses. Neuron 58, 401-413 (2008)

64. Zars, T., Fischer, M., Schulz, R. \& Heisenberg, M. Localization of a short-term memory in Drosophila. Science https://doi.org/10.1126/science.288.5466.672 (2000).

65. Vorobiov, D., Bera, A. K., Keren-Raifman, T., Barzilai, R. \& Dascal, N. Coupling of the muscarinic $\mathrm{m} 2$ receptor to $\mathrm{G}$ protein-activated $\mathrm{K}(+)$ channels via Galpha $(z)$ and a receptor-Galpha $(z)$ fusion protein. Fusion between the receptor and Galpha $(\mathrm{z})$ eliminates catalytic (collision) coupling. J. Biol. Chem. 275, 4166-4170 (2000).

66. Wilson, R. I. \& Laurent, G. Role of GABAergic inhibition in shaping odorevoked spatiotemporal patterns in the Drosophila antennal lobe. J. Neurosci. 25, 9069-9079 (2005).

67. Israel, S., Rozenfeld, E., Weber, D., Huetteroth, W. \& Parnas, M. Two Parallel Pathways Mediate Olfactory-Driven Backward Locomotion. bioRxiv https:// doi.org/10.1101/2020.11.23.393819 (2020).

68. Lerner, H., Rozenfeld, E., Rozenman, B., Huetteroth, W. \& Parnas, M. Differential role for a defined lateral horn neuron subset in naïve odor valence in Drosophila. Sci. Rep. 63169-3 https://doi.org/10.1038/s41598-020-63169-3 (2020).

69. Claridge-Chang, A. et al. Writing memories with light-addressable reinforcement circuitry. Cell 139, 405-415 (2009).

70. Parnas, M., Lin, A. C., Huetteroth, W. \& Miesenböck, G. Odor discrimination in drosophila: from neural population codes to behavior. Neuron 79, 932-944 (2013).

\section{Acknowledgements}

The authors thank Dr. Robert J. Kittel and Dr. Moran Rubinstein for comments on the manuscript. We thank the Bloomington Stock Center and the Vienna Drosophila RNAi Center for fly strains. Molecular graphics and analyses were performed with UCSF Chimera, developed by the Resource for Biocomputing, Visualization, and Informatics at the University of California, San Francisco, with support from NIH P41-GM103311. This work was supported by the Israel Science Foundation (ISF 343/18, MP), the European Research Council (676844, MP), the Deutsche Forschungsgemeinschaft (project number 408264519 to MP) and the Open University of Israel (Internal research grant, YBC).

\section{Author contributions}

E.R.: conceptualization, methodology, investigation, formal analysis, software, writing-review \& editing, visualization. M.T.: investigation, formal analysis. Y.B.C.: investigation, formal analysis, writing-review \& editing, supervision, funding acquisition. M.P.: Initiated the project, conceptualization, methodology, investigation, formal analysis, software, writing-original draft, writing-review \& editing, visualization, supervision, funding acquisition.

\section{Competing interests}

The authors declare no competing interests.

\section{Additional information}

Supplementary information The online version contains supplementary material available at https://doi.org/10.1038/s41467-021-27593-x.

Correspondence and requests for materials should be addressed to Moshe Parnas.

Peer review information Nature Communications thanks Moritz Bunemann and the other, anonymous, reviewer(s) for their contribution to the peer review of this work. Peer reviewer reports are available.

Reprints and permission information is available at http://www.nature.com/reprints

Publisher's note Springer Nature remains neutral with regard to jurisdictional claims in published maps and institutional affiliations.

Open Access This article is licensed under a Creative Commons Attribution 4.0 International License, which permits use, sharing, adaptation, distribution and reproduction in any medium or format, as long as you give appropriate credit to the original author(s) and the source, provide a link to the Creative Commons license, and indicate if changes were made. The images or other third party material in this article are included in the article's Creative Commons license, unless indicated otherwise in a credit line to the material. If material is not included in the article's Creative Commons license and your intended use is not permitted by statutory regulation or exceeds the permitted use, you will need to obtain permission directly from the copyright holder. To view a copy of this license, visit http://creativecommons.org/ licenses/by/4.0\%.

(C) The Author(s) 2021 